\title{
TEM Investigation of Counter-Electrode Structure in Ta Capacitors
}

\author{
P.A. Carvalho*, D. Dias***, A.C. Ferro*, W. Lohwasser** \\ * Departamento de Engenharia de Materiais, Instituto Superior Técnico, Universidade Técnica de \\ Lisboa, Av. Rovisco Pais, 1049-001 Lisboa, Portugal \\ **EPCOS, R. Werner Von Siemens, 7002-504 Évora, Portugal
}

Manganese dioxide experiences growing interest due to new demands in the capacitor and alkaline battery industries. In this work, a pyrolytic manganese dioxide produced by standard solid electrolytic tantalum capacitor technology has been investigated by transmission electron microscopy (TEM). The main purpose of this work was the identification of the $\mathrm{MnO}_{2}$ polymorph adopted, as well as an evaluation of the crystallinity degree and of the size and shape of the crystallites. An assessment of the planar defects present was also carried out, while aiming at a direct observation of microtwinning.

The capacitor microstructure consisted of a Ta skeleton enveloped by the $\mathrm{Ta}_{2} \mathrm{O}_{5}$ dielectric layer and intermediary regions filled with $\mathrm{MnO}_{2}$ grains (Fig.1). The $\mathrm{MnO}_{2}$ counter-electrode was in general well crystallized, with grain sizes typically between 100 and $200 \mathrm{~nm}$. A columnar grain morphology could be observed within $200 \mathrm{~nm}$ from the dielectric. Throughout the counter-electrode layer, the $\mathrm{MnO}_{2}$ grains displayed alternating dark and bright lines due to extensive De Wolff disorder. The structure has been identified by electron microdiffraction as a highly and randomly faulted pyrolusite $\left(\beta-\mathrm{MnO}_{2}\right)$, in which half ramsdellite $\left(\mathrm{R}-\mathrm{MnO}_{2}\right)$ slabs are isolated from each other. As no real intergrowth exists, the structure should be regarded as a faulted pyrolusite rather than nsutite $\left(\gamma-\mathrm{MnO}_{2}\right)$. Planar defects corresponding to two ramsdellite variants coexisted in the same pyrolusite crystallites. No evidence for twinning or microtwinning on $\{011\}$ or $\{031\}$ planes has been found. A powder electron diffraction pattern obtained from the counter electrode layer can be seen in Fig. 2. The intensity profile plotted shows that streaking resulting from De Wolff disorder induces severe peak broadening in the region corresponding to $4.5-3.0 \mathrm{~nm}$.

In De Wolff model [1,2], $\operatorname{Pr}$ is the probability for an arbitrary unit to be a single chain, i.e., $\operatorname{Pr}=1$ for pyrolusite and $\mathrm{P}_{\mathrm{r}}=0$ for ramsdellite (where $\mathrm{r}$ stands for rutile, the prototype structure of $\beta-\mathrm{MnO}_{2}$ ). Furthermore, $P_{R . R}$ is the junction probability of double-chain layers (where $R$ stands for ramsdellite). Therefore, in the present case, $1>\mathrm{P}_{\mathrm{r}}>>0.5$ and $\mathrm{P}_{\mathrm{R} . \mathrm{R}} \approx 0$ as isolated ramsdellite-type faults are present in an essentially rutile-type structure.

The present TEM study enabled to thoroughly characterize the structure of pyrolytic manganese dioxide formed in the particularly restricted environment of a porous Ta body under a typical thermal cycle employed in the Ta capacitor industry. The counter-electrode layer presented 100 to $200 \mathrm{~nm}$ grains of a highly faulted $\beta-\mathrm{MnO}_{2}$ structure, where no microtwinning was present.

\section{References}

[1] De Wolff PM. Acta Cryst 1959;12:341.

[2] Chabre Y, Pannetier J. Prog Solid St Chem 1995;23:1. 


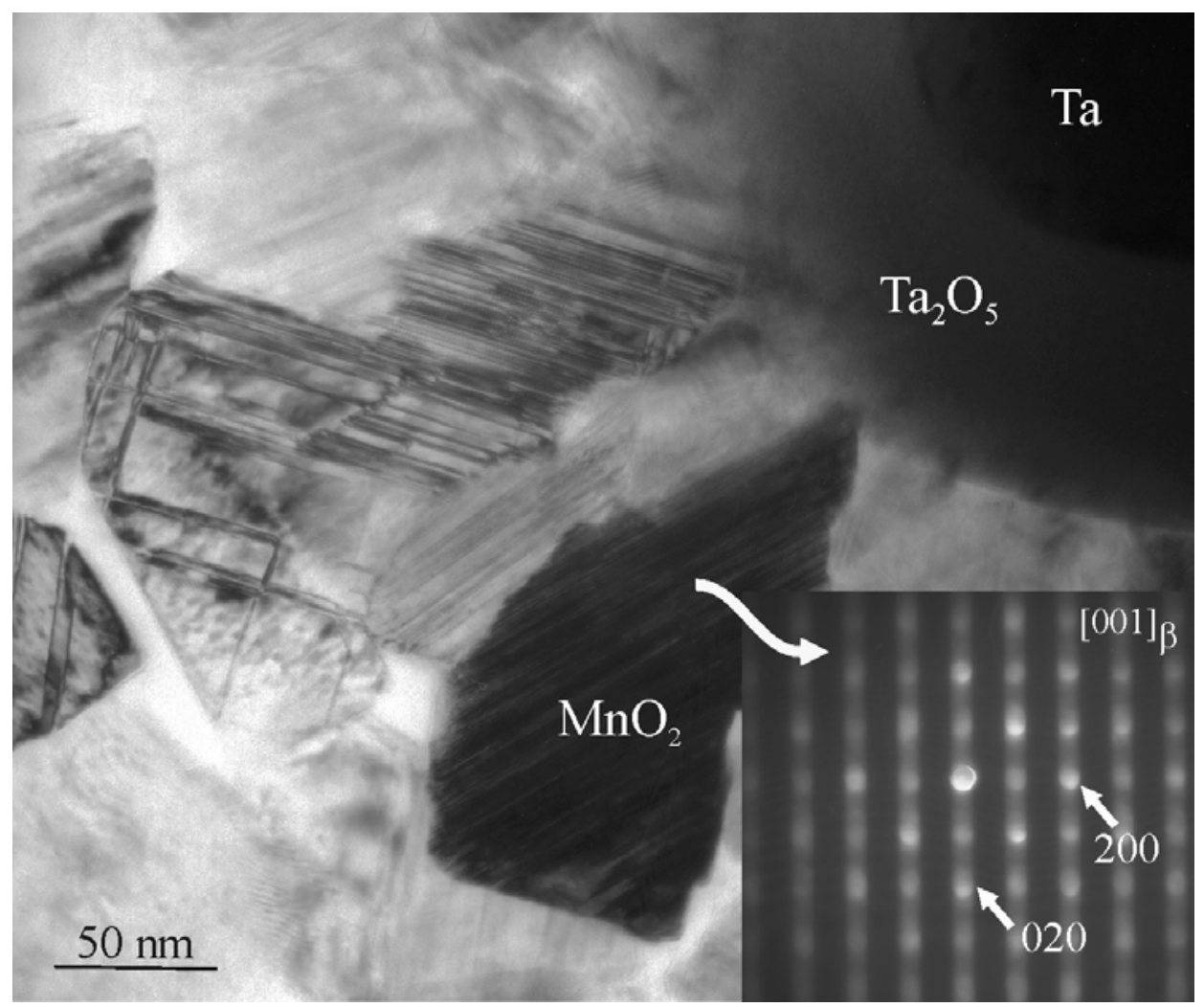

Fig. 1 - Columnar grains of $\mathrm{MnO}_{2}$ around an oxidized tantalum protuberance. A rotated microdiffraction pattern of the strongly diffracting grain is included (streaking is perpendicular to the planar defects).

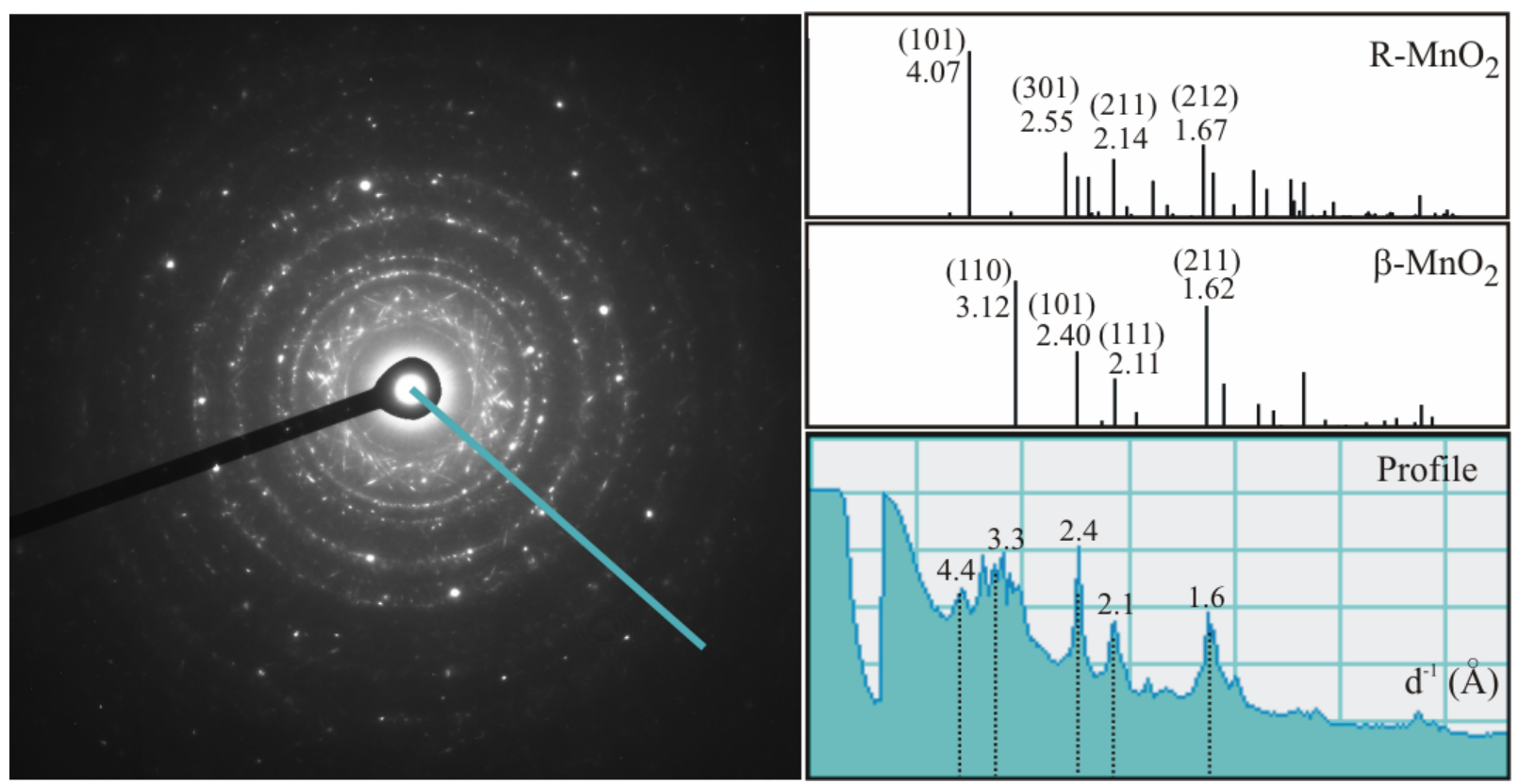

Fig. 2 - Electron powder diffraction pattern of a polycrystalline region in the $\mathrm{MnO}_{2}$ counter-electrode layer. Isolated Ta spots can also be seen. An intensity profile taken across the rings is plotted. Simulated patterns are also shown for comparison. The values refer to interplanar spacings in $\AA$. 